\title{
EFEITO AGUDO DA LIBERAÇ̃̃̃O MIOFASCIAL INSTRUMENTAL NA MODULAÇÃO AUTONÔMICA CARDÍACA EM MULHERES JOVENS PRATICANTES DE MUSCULAÇÃO
}

\author{
Acute effect of instrumental miofascial release on autonomic heart \\ modulation in weight training practicers young women
}

\author{
Ronaldo Henrique Cruvinel Júnior ${ }^{1}$, Ana Paula de Lourdes Pfister ${ }^{1}$, \\ Raiane Luísa Silva Ferreira ${ }^{1}$
}

1-Centro Universitário de Formiga (UNIFOR-MG), Formiga- MG, Brasil.

\begin{abstract}
Resumo
Introdução: A liberação miofascial instrumental é utilizada com a finalidade de liberar aderências do tecido fascial a musculatura esquelética o que pode resultar em alterações do sistema nervoso autônomo cardiovascular tais como a vasodilatação e consequente aumento do fluxo sanguíneo. Objetivo: Avaliar os efeitos da liberação miofascial instrumental na modulação autonômica cardíaca em mulheres jovens praticantes de musculação. Métodos: O estudo foi realizado com 32 mulheres jovens praticantes de musculação, e 15 delas alocadas ao grupo intervenção e 17 ao grupo controle. Foi realizado a técnica de liberação miofascial instrumental com grupo intervenção e foi utilizado o Ultrassom Terapêutico desligado com o grupo controle. A variabilidade da frequência cardíaca foi analisada antes e após realização das técnicas em ambos os grupos. Resultados: A análise de medidas variadas de duas vias Anova Two-Way demonstrou que não houve efeito de tempo (Pré e Pós): SDNN ( $\mathrm{f}=4,71 ; \mathrm{p}=0,27$ ), pNN50\% ( $f=150,1 ; p=0,051), \operatorname{RMSSD}(\mathrm{f}=33,64 ; \mathrm{p}=0,10), \mathrm{LF}(\mathrm{f}=45,35 ; \mathrm{p}=0,09), \mathrm{HF}(\mathrm{f}=19,27 ; \mathrm{p}=0,14)$, $\mathrm{LF} / \mathrm{HF}$ ( $\mathrm{f}=0,30 ; \mathrm{p}=0,29$ ) ou de grupo (Intervenção ou Controle): SDNN ( $\mathrm{f}=0,63 ; \mathrm{p}=0,57), \mathrm{pNN} 50 \%$ $(\mathrm{f}=0,06 ; \mathrm{p}=0,84), \operatorname{RMSSD}(\mathrm{f}=0,04 ; \mathrm{p}=0,87), \mathrm{LF}(\mathrm{f}=55,06 ; \mathrm{p}=0,08), \mathrm{HF}(\mathrm{f}=0,11 ; \mathrm{p}=0,79), \mathrm{LF} / \mathrm{HF}(\mathrm{f}=0,30$; $\mathrm{p}=0,67)$ em todos os índices de variabilidade da frequência cardíaca analisados: Não houve efeito de interação (Grupo x Tempo) pois os grupos apresentaram aumento ou redução no mesmo sentido. Conclusão: $O$ protocolo de liberação miofascial instrumental realizado não influenciou na modulação autonômica cardíaca, pois a partir da análise de estatística inferencial seu efeito não foi superior ao uso do ultrassom terapêutico desligado.
\end{abstract}

Palavras-chave: Fáscia, Sistema Cardiovascular, Sistema Nervoso Autônomo. 


\begin{abstract}
Introduction: Instrumental myofascial release is used to release fascial tissue adhesions to skeletal muscles which may result in changes in the autonomic cardiovascular nervous system such as vasodilation and consequent increase in blood flow. Objective: To evaluate the effects of instrumental myofascial release on cardiac autonomic modulation in weight training practicers young women. Methods: The study was performed with 32 weight training practicers young women, 15 of them being allocated to the intervention group and 17 to the control group. The technique of instrumental myofascial release was performed in the volunteers of the intervention group and the off-set therapeutic ultrasound was used in the volunteers of the control group. Heart rate variability was analyzed before and after performing the techniques in both groups. Results: The analysis of two-way ANOVA two-way showed that there wasn't time effect (Pre and Post): SDNN ( $f=4.71, p=0.27), p N N 50 \%(f=150.1, p=0.05), H F(f=19.27, p=0.14)$, $L F(f=45.35, p=0.09), L F / H F(f=0.30, p=0.29)$ or group (Intervention or Control): $\operatorname{SDNN}(f=0.63$, $p=0.57), p N N 50 \%(f=0.06 ; p=0.84), R M S S D(f=0.04, p=0.87), L F(f=55.06, p=0.08), H F(f=0.11$, $p=0.79), L F / H F(f=0.30, p=0.67)$ in all heart rate variability indexes analyzed: There wasn't interaction effect (Group x Time) since the groups presented increase or decrease in the same direction. Conclusion: The protocol of instrumental myofascial release didn't influence the autonomic cardiac modulation, since from the analysis of inferential statistics its effect wasn't superior to the use of the off-set therapeutic ultrasound.
\end{abstract}

Key words: Fascia, Cardiovascular System, Autonomic Nervous System.

\section{Introdução}

A fáscia é um tecido do tipo conjuntivo que reveste toda a musculatura esquelética do nosso corpo estendendo-se da planta dos pés até o crânio. ${ }^{1} \mathrm{~A}$ liberação miofascial é uma técnica que visa liberar qualquer aderência desse tecido à musculatura, permitindo assim, que o movimento ocorra de forma harmônica em sua amplitude ideal e com a distribuição de forças de maneira correta. ${ }^{2} \mathrm{O}$ sistema miofascial contribui também para funções de propriocepção e



Com relação a prática de exercício físico no Brasil a musculação se tornou a segunda atividade física mais popular, citada por $18,97 \%$ dos praticantes de atividades físicas. Outro dado relativamente importante é que a porcentagem das mulheres que fazem musculação superou a dos homens: a atividade é praticada por $19,56 \%$ das mulheres fisicamente ativas e por $18,46 \%$ dos homens. ${ }^{4}$

A partir dessa ascensão da prática da musculação e devido ao grande número de atletas no Brasil e no mundo a mobilização de tecidos moles utilizando instrumentos tornou-se comum por profissionais da medicina esportiva e fisioterapia. ${ }^{5}$ A realização da técnica de liberação miofascial utilizando instrumentos possui a mesma finalidade da técnica realizada de forma manual, porém oferece vantagem mecânica ao profissional. ${ }^{6,7}$

A eficácia desses tratamentos utilizando a liberação miofascial tem sido observada com base em resultados locais e percepções subjetivas dos pacientes, como a melhoria na amplitude de movimento, escalas analógicas de dor e escala de fadiga percebida. ${ }^{8}$ No entanto já é reconhecido a relação de técnicas de mobilização de tecidos moles e atividade do sistema nervoso autônomo cardiovascular (SNAC), esta relação está associada a vasodilatação e aumento do fluxo sanguíneo o que resulta em diminuição da percepção de dor e aumento da amplitude de movimento. ${ }^{9,10}$

Há indícios na literatura de que a autoliberação miofascial e a massagem terapêutica afetam algumas funções do SNAC como: redução da pressão arterial e frequência cardíaca, aumento dos níveis de endorfinas e variabilidade da frequência cardíaca (VFC), principalmente demonstrando uma maior atividade do sistema parassimpático. ${ }^{11,12,13}$

A variabilidade da frequência cardíaca é um processo fisiológico e representa a variação entre os intervalos R-R de batimentos cardíacos, apontando assim o balanço autonômico entre o sistema simpático e parassimpático. ${ }^{14}$ Alta variabilidade da frequência cardíaca representa uma boa adaptação cardíaca, do contrário a baixa variabilidade da frequência cardíaca é indicativo de uma insuficiência na modulação autonômica cardíaca, uma vez que essa redução pode ser relacionada a estados patológicos e modificações fisiológicas tais como o estresse físico e mental, e no ambiente esportivo essa redução é comumente associada ao estado de excesso de treinamento físico: overtraining. ${ }^{15}$

A análise da VFC pode ser feita através do eletrocardiograma ou utilizando-se um cardiofrequencímetro com objetivo de investigar o funcionamento do SNAC de forma indireta e não invasiva $^{16}$ e pode ser realizada tanto em condições 
patológicas como fisiológicas, inclusive com objetivo de avaliar o efeito de treinamentos e técnicas que podem influenciar o funcionamento do SNAC. ${ }^{17,18}$

Uma questão de extrema relevância desta pesquisa está no fato de compreendermos melhor os mecanismos de ação não locais da técnica de LMI, visto que a mesma é realizada superficialmente sobre a musculatura esquelética, porém o organismo humano é dotado de uma complexidade, e esta deve ser levada em consideração. Uma técnica por mais que aparente ter efeitos apenas no local de sua realização pode influenciar o funcionamento de outros sistemas como o SNAC, levando a repercussões por todo o corpo humano incluindo o sistema cardiovascular que é vital para o funcionamento do sistema musculoesquelético mantendo sua nutrição e adaptação frente a condições impostas.

Partindo desse pressuposto o presente estudo procurou avaliar os efeitos da liberação miofascial instrumental na modulação autonômica cardíaca em mulheres jovens praticantes de musculação.

\section{Metodologia}

Tipo de estudo:

Trata-se de um Ensaio Clínico Randomizado Simples Cego.

\section{Amostra:}

A amostra foi obtida por conveniência, foram incluídas e selecionadas para o estudo 32 mulheres jovens praticantes de musculação com idades entre 18 e 25 anos e que praticavam musculação ao menos três vezes por semana por um período mínimo de seis meses ininterruptos e que no momento da coleta de dados apresentaram sinais vitais como pressão arterial (PA), frequência cardíaca (FC), saturação de oxigênio (StO2) e frequência respiratória (FR) dentro dos padrões de normalidade no momento da coleta de dados. Padrões de normalidade esses considerados como valores entre: $\mathrm{PA}=100 \times 60 \mathrm{mmhg} \mathrm{a}$ $140 \times 90 \mathrm{mmhg}, \mathrm{FC}=60$ a 100 batimentos por minuto (bpm), $\mathrm{StO} 2>90 \%$ e $\mathrm{FR}=16$ a 20 incursões respiratórias por minuto (irpm).

Foram excluídas do estudo aquelas que apresentaram patologias que interferem na variabilidade da frequência cardíaca, tais como: doença pulmonar obstrutiva crônica, diabetes mellitus, hipertensão arterial, tireoidopatias, doenças cardíacas, portadoras de marca-passo, usuários de medicamentos diuréticos e betabloqueadores, voluntárias consideradas obesas pela análise do índice de massa corporal $($ IMC) > que 30, e que apresentaram alguma condição que impedia a realização dos procedimentos de liberação miofascial como: Problemas dermatológicos, lesões na pele, edemas, alterações de coagulação sanguínea e hipersensibilidade vascular, bem como aquelas que não concordaram em assinar o termo de consentimento livre e esclarecido (TCLE),

A randomização foi realizada utilizando envelopes opacos com números de 0 a 100 dentro. As participantes que receberam números ímpares foram alocadas ao grupo intervenção. E aquelas que receberam números pares foram alocadas ao grupo controle-placebo. A amostra foi dividida em dois grupos: Grupo 1 (G1) = Intervenção (liberação miofascial instrumental), Grupo 2 (G2) = ControlePlacebo (toque do ultrassom terapêutico desligado).

Instrumentação e coleta de dados:

O Estudo foi realizado em uma academia do município de Formiga-MG. A coleta de dados foi realizada logo após o treino das voluntárias em uma sala de avaliação com ambiente tranquilo e na presença apenas da voluntária e dois pesquisadores responsáveis pela coleta de dados.

A coleta de dados foi toda realizada no período vespertino das 14:00 ás 17:00 visando anular possíveis alterações hormonais decorrentes do ciclo circadiano.

No dia da coleta de dados as voluntárias realizaram treino da musculatura de Membros Inferiores com duração de 50 minutos, porém não foi controlado a intensidade e volume do treinamento.

Inicialmente foi aplicado o termo de consentimento livre e esclarecido - TCLE, em seguida foi utilizado um formulário de dados sociodemográficos para registrar as variáveis: sociodemográficas, antropométricas e clínicas das voluntárias.

A Aferição dos dados vitais foi feita após a voluntária permanecer por 10 minutos em repouso assentada em uma cadeira. Para mensurar a saturação de oxigênio foi utilizado um oxímetro de pulso portátil modelo Premium da marca Dellamed®. Um esfigmomanômetro e um estetoscópio da marca BIC® modelo Cardiology foram utilizados para aferir a pressão arterial sistêmica. O Frequência cardíaca foi obtida através do uso do frequencímetro Polar® V800. Em seguida as medidas antropométricas foram obtidas com a voluntária descalço, na posição ereta; o peso foi avaliado em balança da marca G.Tech ${ }$, previamente calibrada e a estatura verificada em fita métrica da marca MacroLife ${ }^{\circledR}$. O índice de massa corporal (IMC) foi obtido dividindo-se o peso em quilogramas pelo quadrado da estatura em metros.

Os procedimentos realizados com o $\mathrm{G} 1=$ Intervenção (Liberação Miofascial Instrumental), foram realizados da seguinte forma: As voluntárias permaneceram em repouso em uma maca na posição de decúbito dorsal, para captar os intervalos R-R por um período de cinco minutos para gravação dos índices de VFC, foi utilizado o cardiofrequencímetro modelo V800 da marca Polar® por meio de um cinto elástico fixado no terço inferior do esterno.

Os procedimentos acima mencionados foram realizados antes da técnica de liberação miofascial instrumental. Foi esperado um tempo de cinco minutos após a gravação, para que a cinta torácica do cardiofrequencímetro fosse retirada evitando alterações nos dados. Após retirar a cinta torácica as voluntárias se posicionaram na maca em decúbito ventral de maneira confortável para que a técnica de 
LMI fosse realizada utilizando-se um instrumento de Gua Sha em material acrílico da marca Releaf®.

Foi utilizado também um creme ultradeslizante hipoalergênico da marca Vita Derm®, essa técnica foi realizada por pesquisador previamente treinado e apto para realização da mesma.

A técnica foi realizada por um período de 10 minutos: Tempo esse escolhido devido à técnica de LMI ser comumente realizada por curto período de tempo na prática clínica por profissionais da medicina esportiva e fisioterapia, uma vez que dois minutos a pressão aplicada foi suave com objetivo de dessensibilizar a musculatura e encontrar possíveis aderências, os 8 minutos finais foram aplicados com pressão moderada para que se obtenha os efeitos desejados com a técnica. Essa técnica foi realizada na musculatura posterior do tronco (músculos paravertebrais), devido à proximidade desta musculatura a medula espinhal e nervos espinhais.

Os movimentos foram realizados de forma tridimensional devido a organização do tecido fascial, foram realizados movimentos no sentido paralelo, perpendicular e oblíquo em relação a posição da fibra muscular.

Logo, em seguida as voluntárias retornaram para posição de decúbito dorsal onde foi captado novamente os intervalos R-R por um período de 5 minutos para gravação dos índices de VFC, utilizando o cardiofrequencímetro V800 da marca Polar ${ }^{\circledR}$ por meio de um cinto elástico fixado no terço inferior do esterno.

Os procedimentos realizados com o $\mathrm{G} 2=$ Controle-Placebo: Tratamento simulado (toque do ultrassom terapêutico desligado), seguiram os mesmos princípios metodológicos, porém foi utilizado um aparelho de ultrassom terapêutico modelo Sonopulse III da marca Ibramed®, e também um gel ultradeslizante hipoalergênico da marca Vita Derm®. Esse tratamento simulado foi similar a realização da técnica de LMI: tempo de aplicação, musculatura a ser realizada, e sentido dos movimentos aplicados, apenas a pressão do toque foi menor que a realizada na LMI.

Posteriormente, esses dados foram enviados ao Software Kubios HRV Standard 3.0, para obtenção dos índices de VFC no domínio do tempo e frequência.

Os índices utilizados para análise foram: SDNN: Desvio padrão de todos os intervalos RR normais gravados em um intervalo de tempo, expresso em ms. Reflete a variabilidade da frequência cardíaca de forma global RMSSD: Representa a raiz quadrada da média dos quadrados das diferenças de sucessivos intervalos R-R. Para ser mais fácil, é a média das diferenças entre RR adjacente. Fisiologicamente, RMSSD nos diz sobre as mudanças de intervalos RR consecutivos. Esse parâmetro reflete principalmente a atividade do sistema nervoso parassimpático. pNN50: Porcentagem das diferenças sucessivas entre os intervalos RR que são maiores que $50 \mathrm{~ms}$. Esse parâmetro reflete principalmente a atividade do sistema nervoso parassimpático. HF: Alta frequência durante um intervalo de 0,15-0,40 Hz. Relaciona-se com frequência respiratória e atividade vagal. LF: Baixa frequência correspondente ao intervalo de 0,04 a $0.15 \mathrm{~Hz}$. Sistema nervoso simpático e parassimpático podem estar envolvidos, porém para muitos autores é considerado marcador de ativação simpática e reflete também a função dos barorreceptores. LF/HF: Razão entre as potências $\mathrm{LF} / \mathrm{HF}$, caracteriza o balanço simpático-vagal sobre o coração $(1,5-2,0) .{ }^{19,20,21}$

Análise de dados:

Os dados foram armazenados em planilhas do programa Microsoft Excel 2013 e sistematizados no programa GraphPad Prism versão 5.0. Foi aplicado o teste de Shapiro-Wilk para verificar a normalidade dos dados. Para avaliar as diferenças intergrupais na linha de base, o testes t de Student foi utilizado. A análise de medidas variadas de duas vias Anova Two-Way foi utilizada para analisar os efeitos dos dois tratamentos nos resultados primários em duas avaliações diferentes (dois grupos $\mathrm{x}$ duas vezes). Para descrever os efeitos da intervenções, os coeficientes $d$ de Cohen foram calculados.

Medidas de tendência central e dispersão como média aritmética e desvio-padrão (DP) foram aplicadas para todas as variáveis. O nível de significância adotado para o tratamento estatístico foi de $5 \%(\mathrm{p}<0,05)$. Para melhor visualização dos resultados, estes foram apresentados em gráficos e tabelas.

\section{Cuidados éticos:}

A presente pesquisa somente foi iniciada após a aprovação do Comitê de Ética do Centro Universitário de Formiga (UNIFOR-MG). O protocolo de pesquisa foi inscrito na Plataforma Brasil sobre o número CAAE 87349518.8.0000.5113 no ano de 2018. Após o parecer favorável do Comitê de Ética por meio do parecer de número 2.636.446, foi solicitado aos interessados em participar da pesquisa que assinassem em duas vias o termo de consentimento livre e esclarecido (TCLE). Estas e demais considerações éticas se basearam na resolução 466/12 do Conselho Nacional de Saúde (CNS). O protocolo de pesquisa foi aprovado pelo Registro Brasileiro de Ensaios Clínicos (ReBec) no número RBR-8shb3h.

\section{Resultados}

Um total de 32 voluntárias foram recrutadas para o estudo, 15 delas foram alocadas ao grupo intervenção e 17 ao grupo controle. Os grupos se demonstraram homogêneos nas variáveis: Idade, IMC, pressão arterial, frequência cardíaca, frequência respiratória, saturação de oxigênio e minutos de musculação realizados por semana. Houve diferença significativa apenas nas variáveis peso e altura. Com relação ao índice de massa corporal (IMC) a média de ambos os grupos estava dentro da classificação de peso ideal para população adulta (Tabela 1). 
TABELA 1 - Informações sociodemográficas, antropométricas e clínicas dos grupos intervenção e controle.

Grupo 1 - Intervenção (Liberação

Miofascial Instrumental)
Grupo 2 - Controle Placebo (Ultrasson

Terapêutico Desligado)

\section{Características}

$(n=15)$

Idade (anos)

Altura (m)

IMC

(Peso/Altura ${ }^{2}$ )

PA Sistólica

(mmhg)

PA Diastólica

(mmhg)

FC (bpm)

StO2 (\%)

R (irpm)

Minutos de

musculação por

semana

Tempo

Musculação em

meses

\author{
Média \pm Desvio \\ padrão
}

$20,60 \pm 1,78$

$56,04 \pm 6,19$

$1,62 \pm 0,04$

$21,29 \pm 1,83$

$119,33 \pm 12,89$

$75,33 \pm 10,87$

$93,80 \pm 7,23$

$97,20 \pm 1,04$

$18,13 \pm 3,13$

$208,00 \pm 43,08$

$26,06 \pm 21,48$

$$
26,06 \pm 21,48
$$

\section{Características \\ $(n=17)$}

Idade (anos)

Peso (Kg)

Altura (m)

IMC

(Peso/Altura ${ }^{2}$ )

PA Sistólica

(mmhg)

PA Diastólica

(mmhg)

FC (bpm)

$\mathrm{StO} 2(\%)$

FR (irpm)

Minutos de musculação por semana

Tempo

Musculação em

meses
$13,58 \pm 10,28$

Valor de p

(IC95\%)

0,16

$0,03 *$

$0,02 *$

0,06

$23,64 \pm 3,67$

$115,29 \pm 10,91$

0,41

$70,58 \pm 8,72$

0,16

$93,94 \pm 8,06$

$97,05 \pm 0,72$

$18,47 \pm 2,11$

0,72

$229,41 \pm 47,08$

0,10

Legenda: n: população. Kg: Kilogramas. m: metros. IMC: Índice de Massa Corporal. PA: Pressão Arterial. mmhg: milímetros de mercúrio. FC: Frequência Cardíaca. Bpm: Batimentos por minuto. StO2: saturação de oxigênio. FR: Frequência respiratória. irpm: Incursões respiratórias por minuto. \%: porcentagem. IC: Intervalo de Confiança.

Fonte: O autor, 2018.

Com relação aos valores médios dos índices de variabilidade da frequência cardíaca dos grupos intervenção e controle em repouso, estes se demonstraram pareados em todos os índices (Tabela 2). 
TABELA 2 - Média dos índices de variabilidade da frequência cardíaca de ambos os grupos em repouso.

Grupo 1= Intervenção (Liberação Miofascial Instrumental)

\section{Grupo 2= Controle-Placebo: (Toque do}

Ultrasson Terapêutico desligado)

\begin{tabular}{|c|c|c|c|c|}
\hline Características $(n=15)$ & $\begin{array}{c}\text { Média } \pm \text { Desvio } \\
\text { padrão }\end{array}$ & Características $(n=17)$ & $\begin{array}{c}\text { Média } \pm \text { Desvio } \\
\text { padrão }\end{array}$ & $\begin{array}{c}\text { Valor de p } \\
(\text { IC95\%) }\end{array}$ \\
\hline SDNN (ms) & $37,40 \pm 13,41$ & SDNN (ms) & $38,48 \pm 21,86$ & 0,85 \\
\hline pNN50\% & $7,90 \pm 9,68$ & pNN50\% & $8,40 \pm 10,95$ & 0,74 \\
\hline RMSSD (ms) & $27,33 \pm 12,65$ & RMSSD (ms) & $28,67 \pm 18,64$ & 0,89 \\
\hline $\mathrm{LF}(\mathrm{ms} 2)$ & $1256,53 \pm 1306,14$ & $\mathrm{LF}(\mathrm{ms} 2)$ & $977,82 \pm 841,00$ & 0,30 \\
\hline $\mathrm{HF}(\mathrm{ms} 2)$ & $313,33 \pm 241,15$ & $\mathrm{HF}(\mathrm{ms} 2)$ & $387,88 \pm 408,48$ & 0,88 \\
\hline $\mathrm{LF} / \mathrm{HF}$ & $3,97 \pm 4,43$ & $\mathrm{LF} / \mathrm{HF}$ & $3,59 \pm 2,07$ & 0,32 \\
\hline
\end{tabular}

Legenda: n: população. SDNN: Desvio padrão de todos os intervalos RR normais gravados em um intervalo de tempo RMSSD: Raiz quadrada da média dos quadrados das diferenças de sucessivos intervalos R-R. pNN50: Porcentagem das diferenças sucessivas entre os intervalos RR que são maiores que $50 \mathrm{~ms}$. HF: Alta Frequência durante um intervalo de 0,15-0,40 Hz. LF: Baixa frequência correspondente ao intervalo de 0,04 a $0.15 \mathrm{~Hz}$. LF/HF: Razão entre as potências LF/HF ms: milissegundos. ms2: milissegundos ao quadrado. \%: porcentagem. IC: Intervalo de Confiança.

Fonte: $\mathrm{O}$ autor, 2018.

A análise de medidas variadas de duas vias Anova Two-Way demonstrou que não houve efeito de tempo (Pré e Pós): SDNN (f=4,71; p=0,27), pNN50\% $(\mathrm{f}=150,1 ; \mathrm{p}=0,051), \mathrm{RMSSD}(\mathrm{f}=33,64 ; \mathrm{p}=0,10), \mathrm{LF}$ $(\mathrm{f}=45,35 ; \mathrm{p}=0,09), \quad \mathrm{HF}(\mathrm{f}=19,27 ; \mathrm{p}=0,14), \mathrm{LF} / \mathrm{HF}$ $(\mathrm{f}=0,30 ; \mathrm{p}=0,29)$ ou de grupo (Intervenção ou Controle): $\operatorname{SDNN}(\mathrm{f}=0,63 ; \mathrm{p}=0,57), \mathrm{pNN} 50 \%(\mathrm{f}=0,06$; $\mathrm{p}=0,84), \quad \operatorname{RMSSD}(\mathrm{f}=0,04 ; \mathrm{p}=0,87), \mathrm{LF} \quad(\mathrm{f}=55,06$; $\mathrm{p}=0,08), \operatorname{HF}(\mathrm{f}=0,11 ; \mathrm{p}=0,79), \mathrm{LF} / \mathrm{HF}(\mathrm{f}=0,30 ; \mathrm{p}=0,67)$ em todos os índices de variabilidade da frequência cardíaca analisados: Não houve efeito de interação (Grupo $\mathrm{x}$ Tempo) pois os grupos apresentaram aumento ou redução no mesmo sentido (Figura 1).
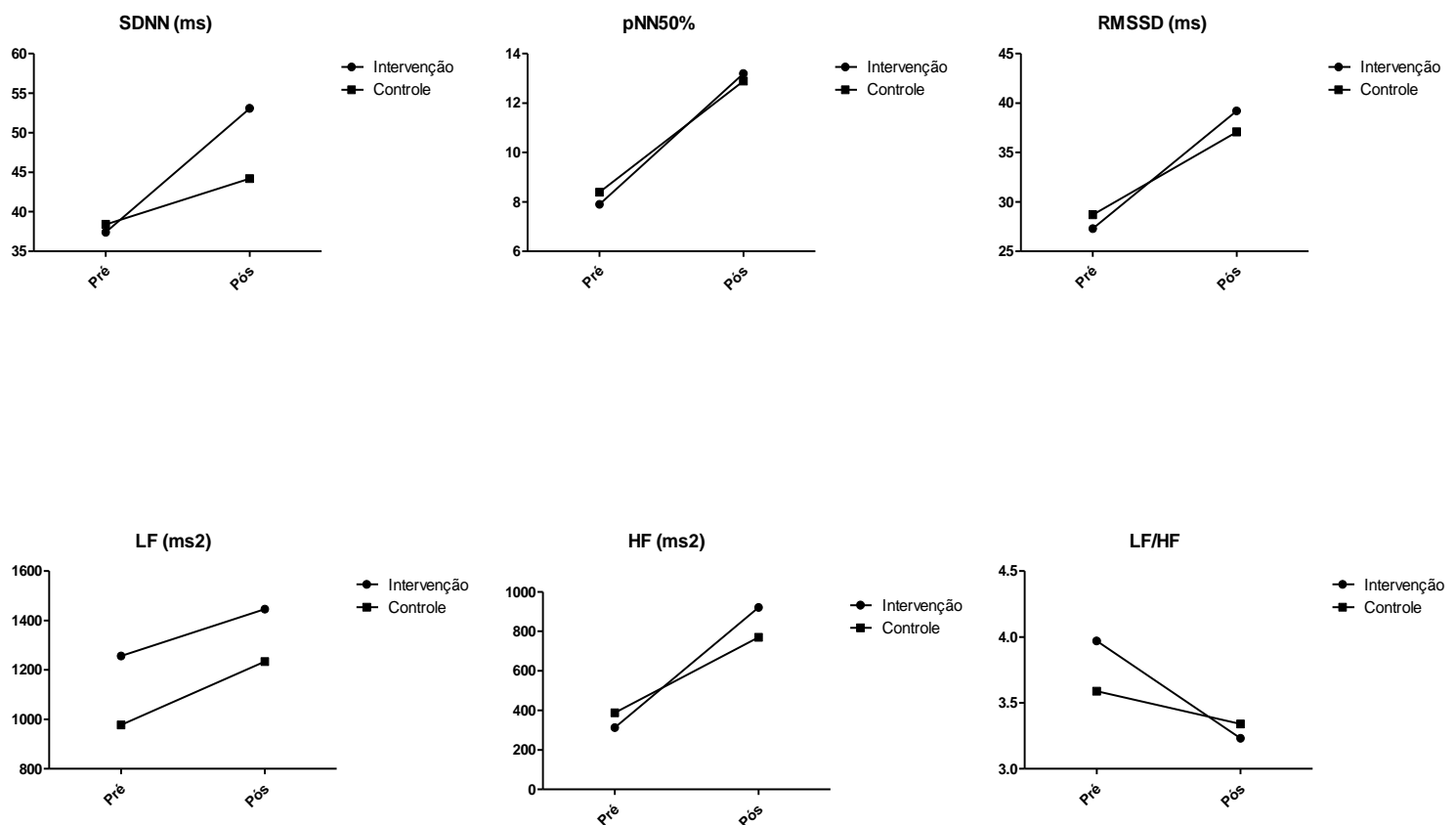

FIGURA 1: A,B,C,D,E,F) Média de diferenças de resultados primários e diferenças de resultados intergrupais demonstrando efeito. A barra de desvio padronizada mostra a variabilidade dos resultados. LEGENDA: SDNN: Desvio padrão de todos os intervalos RR normais gravados em um intervalo de tempo RMSSD: Raiz quadrada da média dos quadrados das diferenças de sucessivos intervalos R-R. pNN50: Porcentagem das diferenças sucessivas entre os intervalos RR que são maiores que 50 ms. HF: Alta Frequência durante um intervalo de 0,15-0,40 Hz. LF: Baixa frequência correspondente ao intervalo de 0,04 a 0.15Hz. LF/HF: Razão entre as potências LF/HF ms: milissegundos. ms2: milissegundos ao quadrado. \%: porcentagem.

Fonte: $\mathrm{O}$ autor, 2018. 
Os resultados demonstraram que não houve efeito de tempo em todos os índices de variabilidade da frequência cardíaca analisados (Tabela 3).

TABELA 3 - Estatística descritiva e inferencial da mensuração do efeito sobre os resultados primários de ambos os grupos

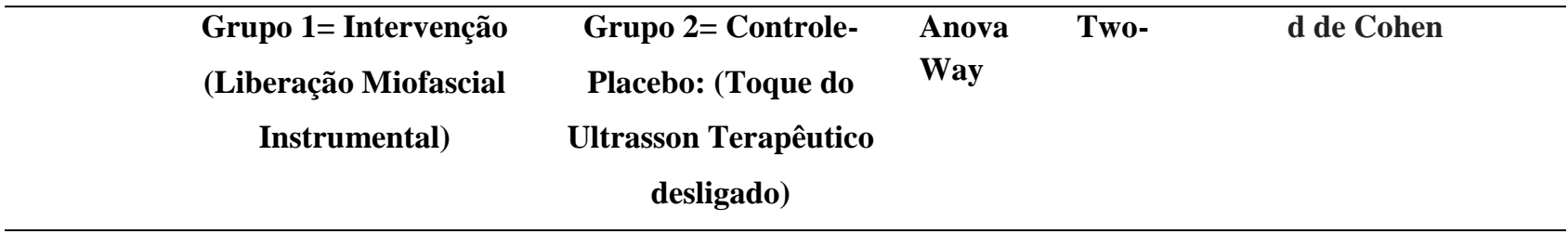

\begin{tabular}{|c|c|c|c|c|c|c|c|}
\hline & Pré (a) & Pós (b) & Pré (c) & Pós (d) & Efeito & de tempo & Tamanho efeito (d d \\
\hline $\begin{array}{l}\text { Índices de } \\
\text { VFC }\end{array}$ & Média (DP) & Média (DP) & Média (DP) & $\begin{array}{l}\text { Média } \\
\text { (DP) }\end{array}$ & $\mathrm{F}$ & $\begin{array}{c}\mathrm{p} \\
(\mathrm{IC} 95 \%)\end{array}$ & d Cohen (efeito) \\
\hline $\begin{array}{l}\text { SDNN } \\
(\mathrm{ms})\end{array}$ & $\begin{array}{c}37,40 \\
(13,41)\end{array}$ & $\begin{array}{c}53,13 \\
(21,12)\end{array}$ & $\begin{array}{c}38,48 \\
(21,86)\end{array}$ & $\begin{array}{c}44,26 \\
(16,79)\end{array}$ & 4,71 & 0,27 & $\begin{array}{c}0.22 \text { (Pequeno) }^{\mathrm{b}, \mathrm{d}} \\
0.40{\text { (Médio })^{\mathrm{a}, \mathrm{b}}} \\
0,14 \text { (Pequeno }^{\mathrm{c}, \mathrm{d}}\end{array}$ \\
\hline pNN50\% & $\begin{array}{c}7,90 \\
(9,68)\end{array}$ & $\begin{array}{c}13,20 \\
(12,58)\end{array}$ & $\begin{array}{c}8,40 \\
(10,95)\end{array}$ & $\begin{array}{c}12,90 \\
(12,35)\end{array}$ & 150,1 & 0,051 & $\begin{array}{l}0.01 \text { (Pequeno }^{\mathrm{b}, \mathrm{d}} \\
0.22 \text { (Pequeno }^{\mathrm{a}, \mathrm{b}} \\
0.18 \text { (Pequeno }^{\mathrm{c}, \mathrm{d}}\end{array}$ \\
\hline $\begin{array}{l}\text { RMSSD } \\
(\mathrm{ms})\end{array}$ & $\begin{array}{c}27,33 \\
(12,65)\end{array}$ & $\begin{array}{c}39,24 \\
(14,91)\end{array}$ & $\begin{array}{c}28,67 \\
(18,64)\end{array}$ & $\begin{array}{c}37,08 \\
(19,02)\end{array}$ & 33,64 & 0,10 & $\begin{array}{c}0.06 \text { (Pequeno) }^{\mathrm{b}, \mathrm{d}} \\
0.39 \text { (Médio) }^{\mathrm{a}, \mathrm{b}} \\
0.21 \text { (Pequeno) }^{\mathrm{c}, \mathrm{d}}\end{array}$ \\
\hline $\mathrm{LF}(\mathrm{ms} 2)$ & $\begin{array}{c}1256,53 \\
(1306,14)\end{array}$ & $\begin{array}{c}1446,33 \\
(1424,70)\end{array}$ & $\begin{array}{c}977,82 \\
(841,00)\end{array}$ & $\begin{array}{l}1233,88 \\
(800,74)\end{array}$ & 45,35 & 0,09 & $\begin{array}{l}0.09 \text { (Pequeno }^{\mathrm{b}, \mathrm{d}} \\
0.06 \text { (Pequeno }^{\mathrm{a}, \mathrm{b}} \\
0.15 \text { (Pequeno }^{\mathrm{c}, \mathrm{d}}\end{array}$ \\
\hline $\mathrm{HF}(\mathrm{ms} 2)$ & $\begin{array}{c}313,33 \\
(241,15)\end{array}$ & $\begin{array}{c}921,33 \\
(836,56)\end{array}$ & $\begin{array}{c}387,88 \\
(408,48)\end{array}$ & $\begin{array}{c}770,35 \\
(655,11)\end{array}$ & 19,27 & 0,14 & $\begin{array}{c}0.09 \text { (Pequeno) }^{\mathrm{b}, \mathrm{d}} \\
0.44 \text { (Médio) }^{\mathrm{a}, \mathrm{b}} \\
0.33 \text { (Pequeno) }^{\mathrm{c}, \mathrm{d}}\end{array}$ \\
\hline $\mathrm{LF} / \mathrm{HF}$ & $\begin{array}{r}3,97 \\
(4,43)\end{array}$ & $\begin{array}{l}3,23 \\
(1,40)\end{array}$ & $\begin{array}{c}3,59 \\
(2,07)\end{array}$ & $\begin{array}{c}3,34 \\
(3,87)\end{array}$ & 0,30 & 0,29 & $\begin{array}{l}0.01 \text { (Pequeno }^{\mathrm{b}, \mathrm{d}} \\
0.11 \text { (Pequeno }^{\mathrm{a}, \mathrm{b}} \\
0.04 \text { (Pequeno }^{\mathrm{c}, \mathrm{d}}\end{array}$ \\
\hline
\end{tabular}

Legenda: n: população. SDNN: Desvio padrão de todos os intervalos RR normais gravados em um intervalo de tempo RMSSD: Raiz quadrada da média dos quadrados das diferenças de sucessivos intervalos R-R. pNN50: Porcentagem das diferenças sucessivas entre os intervalos RR que são maiores que $50 \mathrm{~ms}$. HF: Alta Frequência durante um intervalo de 0,15-0,40 Hz. LF: Baixa frequência correspondente ao intervalo de 0,04 a $0.15 \mathrm{~Hz}$. LF/HF: Razão entre as potências LF/HF ms: milissegundos. ms2: milissegundos ao quadrado. \%: porcentagem. DP: Desvio Padrão. IC: Intervalo de Confiança. F: Efeito. *Significância estatística.

Fonte: O autor, 2018.

\section{Discussão}

O presente estudo avaliou os efeitos da liberação miofascial instrumental na modulação autonômica cardíaca em mulheres jovens praticantes de musculação. A partir dos resultados ficou evidenciado que não houve diferença significativa quando comparados os índices de VFC entre os grupos intervenção e controle, bem como os valores pré e pós intervenções quando utilizado a estatística inferencial para análise dos resultados.

O delineamento do presente estudo baseou-se em um editorial publicado no Brazilian Journal of Physical Therapy, que descreve a importância de se determinar o significado clínico dos resultados da 
pesquisa clínica em fisioterapia, pois a realização de um teste de hipótese, não fornece informações acerca da importância clínica dos resultados de pesquisas na área da saúde. Levando em conta as limitações de significância estatística, é de suma importância a realização de testes de efeito e que os resultados dessas pesquisas sejam analisados tendo em vista a relevância clínica dos resultados. ${ }^{22}$

Os achados do presente estudo não corroboram com o estudo de Arroyo-Morales et al. ${ }^{23}$ em que foram avaliados os efeitos da liberação miofascial (LM) após um protocolo de exercício de alta intensidade. O grupo placebo apresentou menor valor de $\mathrm{HF}$ e maior valor no índice LF/HF após o tratamento simulado quando comparado ao grupo intervenção, indicando um atraso no processo de recuperação.

Vale ressaltar a disparidade metodológica encontrada no estudo de Arroyo-Morales et al. ${ }^{23}$ que incluiu voluntários homens em sua pesquisa, realizou protocolo de liberação miofascial manual com duração de 40 minutos, ou seja, superior ao presente estudo, e a análise da VFC foi realizada por meio de gravação holter. Estudo realizado por Kingsley et al. ${ }^{24}$ afirma que monitores da marca Polar apresentam alta correlação com a análise dos índices de VFC realizados por eletrocardiograma.

Delaney et al. ${ }^{25}$ em estudo semelhante, avaliou os efeitos a curto prazo da liberação miofascial em pontos gatilhos sobre a modulação autonômica cardíaca de indivíduos saudáveis de ambos os sexos. O grupo intervenção apresentou valores superiores nos seguintes índices após intervenção: SDNN, RMSSD, pNN50\%, HF, e uma redução de LF/HF, o que sugere uma maior ativação parassimpática em comparação ao grupo controle. Da mesma forma esses resultados não corroboram com os achados do presente estudo, talvez isto se deva ao fato de os autores incluírem homens em sua pesquisa, bem como realizarem e técnica por tempo de 20 minutos em pontos gatilhos, ou seja, uma condição patológica além do fato de ter sido realizada em musculatura de trapézio superior, músculos subocciptais e esternocleidomastoideo, o que difere dos procedimentos adotados na presente pesquisa.

Em outro estudo realizado por Henley et al. ${ }^{26}$, foi avaliado a relação do tratamento manipulativo osteopático com a atividade do sistema nervoso autônomo de indivíduos saudáveis de ambos os sexos. Quando os indivíduos receberam liberação miofascial estes apresentaram uma maior redução do índice $\mathrm{LF} / \mathrm{HF}$, e aumento do HF o que sugere uma maior atividade parassimpática. Esses achados não corroboram com o presente estudo, talvez se deva ao fato de os pesquisadores realizarem protocolo com técnicas diversas de mobilização de tecidos moles utilizadas na osteopatia, além do fato de realizarem o protocolo em musculatura cervical e por tempo de 20 minutos. Outro ponto que exerce influência sobre os resultados está no fato de os participantes terem realizados sessões com intervalo de $24 \mathrm{~h}$ pois os participantes atuaram como seus próprios controles.
O mecanismo o qual medeia as respostas cardiovasculares após manobras terapêuticas miofasciais ainda precisa ser melhor descrito, porém sabe-se que um grupo específico de mecanorreceptores são estimulados por essas manobras, principalmente as que envolvem pressão e tração, desencadeando uma resposta predominantemente parassimpática na modulação autonômica cardíaca. ${ }^{27}$

Por meio do presente estudo é possível afirmar que a liberação miofascial instrumental quando realizada por curto período de tempo não exerce influência significativa na modulação autonômica cardíaca em mulheres jovens praticantes de musculação. Entretanto os estudos encontrados na literatura e acima citados contradizem os achados do presente estudo e afirmam que a liberação miofascial influencia na modulação autonômica cardíaca de modo a aumentar a atividade parassimpática o que corrobora com uma melhor recuperação do sistema cardiovascular pós exercício. Porém é imprudente generalizar estes achados de uma população saudável para aqueles com processos de doença.

Diante do exposto, esclarece-se, ainda, que o presente estudo apresenta algumas limitações que devem ser levadas em conta na sua análise. Não foi delimitado um protocolo específico de exercícios, nem mesmo controlado sua intensidade e volume. Outra limitação encontra-se no fato de a técnica ter sido realizada por um curto período de tempo e foram avaliados apenas os efeitos agudos imediatamente após a realização da técnica de LMI.

Assim, este estudo é de suma importância e contribui de maneira significativa norteando parâmetros e fornecendo informações aos profissionais da medicina esportiva acerca dos efeitos não locais da LMI, aos quais não influenciaram positivamente na recuperação pós exercício normalizando a atividade cardiovascular, principalmente pelo aumento não significativo da atuação do sistema nervoso autônomo parassimpático.

$\mathrm{Na}$ tentativa de se obter mais informações fidedignas sobre os efeitos da LMI sobre o funcionamento do sistema nervoso autônomo bem como de variáveis fisiológicas relacionadas a aptidão e treinamento físico, sugere-se que novos estudos sejam realizados com maiores amostras, e que realizem análises tais como: Níveis de cortisol, testosterona, lactato e citocinas inflamatórias. Sugerese também que em estudos futuros sejam avaliados efeitos da LMI a longo prazo e com período de followup.

Sugere-se também que novos estudos sejam realizados com objetivos semelhantes aos deste, para que, assim, os profissionais da área de saúde tenham cada vez mais parâmetros confiáveis para realizar a técnica de LMI e, ainda, que esta possa também ser realizada em pessoas com determinadas patologias que comprometam sua modulação autonômica cardíaca. 


\section{Conclusão}

Este estudo identificou que o protocolo de liberação miofascial instrumental realizado não influenciou na modulação autonômica cardíaca. Assim, essa técnica não se demonstrou eficaz pois seus efeitos no sistema nervoso autônomo não foram superiores ao uso do ultrassom terapêutico desligado na recuperação pós-exercício na tentativa de normalizar a atividade cardiovascular, principalmente por não ter sido capaz de aumentar a atuação do sistema nervoso autônomo parassimpático de maneira significativa.

\section{Declaração de conflitos de interesses}

Os autores do artigo afirmam que não houve nenhuma situação de conflito de interesse, tais como propostas de financiamento, emissão de pareceres, promoções ou participação em comitês consultivos ou diretivos, entre outras, que pudessem influenciar no desenvolvimento do trabalho.

\section{Agradecimentos}

Os autores agradecem a FAPEMIG pelo financiamento do projeto.

\section{Referências}

1- RICHMAN, E. D; TYO, B. M; NICKS, C. R. Combined Effects of Self-Myofascial Release and Dynamic Stretching on Range of Motion, Jump, Sprint, and Agility Performance. Journal of strength and conditioning research, 2018.

2- PAVAN, P. G; PACHERA, P; FORESTIERO, A; NATALI, A. N. Investigation of interaction phenomena between crural fascia and muscles by using a three-dimensional numerical model. Medical \& biological engineering \& computing, v. 55, n. 9, p. 1683-1691, 2017.

3- HUIJING, P. A; BAAN, G. C. Myofascial force transmission via extramuscular pathways occurs between antagonistic muscles. Cells Tissues Organs, v. 188, n. 4, p. 400-414, 2008.

4- DE SA, T. H; GARCIA, L. M. T; CLARO, R. M. Frequency, distribution and time trends of types of leisure-time physical activity in Brazil, 20062012. International journal of public health, v. 59, n. 6, p. 975-982, 2014.

5- MACDONALD, N; BAKER, R; CHEATHAM, S. $\mathrm{W}$. The effects of instrument assisted soft tissue mobilization on lower extremity muscle performance: a randomized controlled trial. International journal of sports physical therapy, v. 11, n. 7, p. 1040, 2016. 6- KIM, J; SUNG, D. J; LEE, J. Therapeutic effectiveness of instrument-assisted soft tissue mobilization for soft tissue injury: mechanisms and practical application. Journal of exercise rehabilitation, v. 13, n. 1, p. 12, 2017.
7- MARKOVIC, G. Acute effects of instrument assisted soft tissue mobilization vs. foam rolling on knee and hip range of motion in soccer players. Journal of bodywork and movement therapies, v. 19, n. 4, p. 690-696, 2015.

8- WEERAPONG, P; HUME, P. A; KOLT, G. S. The mechanisms of massage and effects on performance, muscle recovery and injury prevention. Sports medicine, v. 35, n. 3, p. 235-256, 2005.

9- RODRÍGUEZ-HUGUET, M; GIL-SALÚ, J. L; RODRÍGUEZ-HUGUET, P; CABRERA-AFONSO, J. R; LOMAS-VEGA, R. Effects of myofascial release on pressure pain thresholds in patients with neck pain: a single-blind randomized controlled trial. American journal of physical medicine $\&$ rehabilitation, v. 97 , n. 1, p. 16-22, 2018.

10- BEARDSLEY, C; ŠKARABOT, J. Effects of selfmyofascial release: a systematic review. Journal of bodywork and movement therapies, v. 19, n. 4, p. 747-758, 2015.

11- KIM, K; PARK, S; GOO, B. O; CHOI, S. C. Effect of self-myofascial release on reduction of physical stress: a pilot study. Journal of physical therapy science, v. 26, n. 11, p. 1779-1781, 2014.

12- CHAN, Y. C; WANG, T. J; CHANG, C. C; CHEN, L. C; CHU, H. Y; LIN, S. P; CHANG, S. T. Short-term effects of self-massage combined with home exercise on pain, daily activity, and autonomic function in patients with myofascial pain dysfunction syndrome. Journal of physical therapy science, v. 27, n. 1, p. 217-221, 2015.

13- LIMA-BORGES, D. S; MARTINEZ, P. F; VANDERLEI, L. C. M; BARBOSA, F. S; OLIVEIRA-JUNIOR, S. A. Autonomic modulations of heart rate variability are associated with sports injury incidence in sprint swimmers. The Physician and sportsmedicine, p. 1-11, 2018.

14- SABOUL, D; PIALOUX, V; HAUTIER, C. The breathing effect of the $\mathrm{LF} / \mathrm{HF}$ ratio in the heart rate variability measurements of athletes. European journal of sport science, v. 14, n. sup1, p. S282-S288, 2014.

15- LOMBARDI, F; MALLIANI, A; PAGANI, M; CERUTTI, S. Heart rate variability and its sympathovagal modulation. Cardiovascular research, v. 32, n. 2, p. 208-216, 1996.

16- CHASWAL, M; KAPOOR, R; BATRA, A; VERMA, S; YADAV, B. S. Heart Rate Variability and Cardiovascular Reflex Tests for Assessment of Autonomic Functions in Preeclampsia. International journal of hypertension, v. 2018, 2018.

17- SOLANA-TRAMUNT, M; MORALES, J; BUSCÀ, B; CARBONELL, M; RODRÍGUEZZAMORA, L. Heart Rate Variability in Elite Synchronized Swimmers. International journal of sports physiology and performance, p. 1-24, 2018. 18- WEIPPERT, M; BEHRENS, M; MAUMOELLER, A; BRUHN, S; Behrens, K. Relationship between morning heart rate variability and creatine kinase response during intensified training in 
recreational endurance athletes. Frontiers in physiology, v. 9, 2018.

19- MARÃES, V. R. F. S. Frequência cardíaca e sua variabilidade: análises e aplicações. Revista andaluza de Medicina del Deporte, v. 3, n. 1, 2010.

20- MALIK, M; BIGGER, J. T; CAMM, A. J; KLEIGER, R. E; MALLIANI, A; MOSS, A. J; SCHWARTZ, P. J. Heart rate variability: Standards of measurement, physiological interpretation, and clinical use. European heart journal, v. 17, n. 3, p. 354-381, 1996.

21- VANDERLEI, L. C. M., PASTRE, C. M; HOSHI, R. A; CARVALHO, T. D. D; GODOY, M. F. D. Basic notions of heart rate variability and its clinical applicability. Brazilian Journal of Cardiovascular Surgery, v. 24, n. 2, p. 205-217, 2009.

22- ARMIJO-OLIVO, S. The importance of determining the clinical significance of research results in physical therapy clinical research. Brazilian journal of physical therapy, v. 22, n. 3, p. 175, 2018. 23- ARROYO-MORALES, M; OLEA, N; MARTINEZ, M; MORENO-LORENZO, C; DAZRODRGUEZ, L; HIDALGO-LOZANO, A. Effects of myofascial release after high-intensity exercise: a randomized clinical trial. Journal of manipulative and physiological therapeutics, v. 31, n. 3, p. 217223, 2008.

24- KINGSLEY, M; LEWIS, M. J; MARSON, R. E. Comparison of polar $810 \mathrm{~s}$ and an ambulatory ECG system for RR interval measurement during progressive exercise. International journal of sports medicine, v. 26, n. 01, p. 39-44, 2005.

25- DELANEY, J. P; LEONG, K. S; WATKINS, A; BRODIE, D. The short-term effects of myofascial trigger point massage therapy on cardiac autonomic tone in healthy subjects. Journal of advanced nursing, v. 37, n. 4, p. 364-371, 2002.

26- HENLEY, C. E; IVINS, D; MILLS, M; WEN, F. K; BENJAMIN, B. A. Osteopathic manipulative treatment and its relationship to autonomic nervous system activity as demonstrated by heart rate variability: a repeated measures study. Osteopathic Medicine and Primary Care, v. 2, n. 1, p. 7, 2008.

27- SATO, Y; SCHAIBLE, H-G; SCHMIDT, R. F. Types of afferents from the knee joint evoking sympathetic reflexes in cat inferior cardiac nerves. Neuroscience letters, v. 39, n. 1, p. 71-75, 1983. 